\title{
CP violation in $b$-hadron decays using top-pair events in 8 TeV ATLAS data
}

\author{
Adelina D'Onofrio*t \\ Queen Mary University of London \\ E-mail: adelina.donofrio@gmul.ac.uk
}

\begin{abstract}
The large number of top-antitop pair events produced in $p p$ colllisions at the Large Hadron Collider (LHC) provides a unique source of $b$-quarks that can be used to probe the CP violation in heavy-flavour mixing and decay. This measurement focuses on semileptonic top-antitop events where one of the $W$-bosons decays hadronically and the other one decays leptonically. The charge of the lepton (electron or muon) from the $W$-boson tags the charge of the $b$-quark at production. In events where a muon is associated to the semileptonic decay of the $b$-quark (either directly or after a $b \rightarrow c$ hadronic transition), two charge asymmetries (CA) and several CP asymmetries, based on the charges of the lepton from the $W$-boson and this muon, can be measured. The first measurement of the $\mathrm{CA}$ and $\mathrm{CP}$ asymmetries in heavy flavour $b$ - or $c$-decays from top-antitop lepton+jets events is presented using the data collected with the ATLAS detector during Run 1 of the LHC. An overview of the future perspectives and improvements of this measurement in Run 2 is also provided.
\end{abstract}

The European Physical Society Conference on High Energy Physics

5-12 July, 2017

Venice

\footnotetext{
* Speaker.

${ }^{\dagger}$ On behalf of the ATLAS Collaboration. Thanks to my Supervisor and all the NPTEV-TQP2020 group.
} 

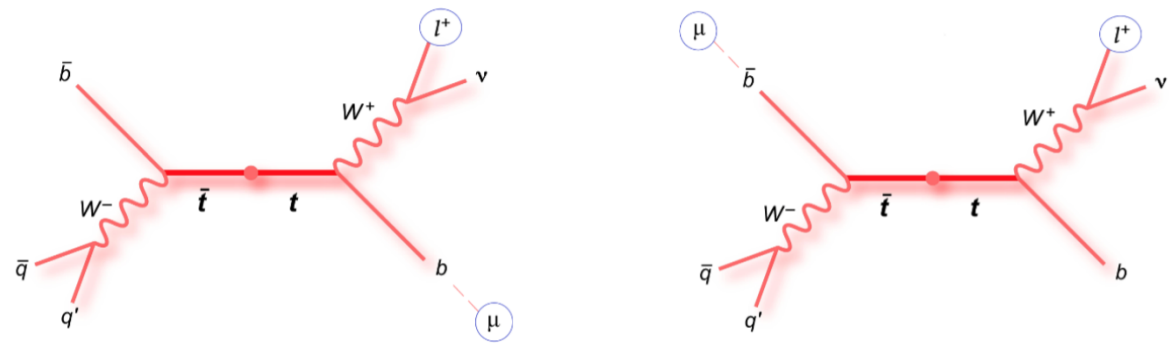

Figure 1: Illustration of same- and different-top SMT muons.

\section{Analysis overview and CPV observables}

$\mathrm{CP}$ violation in weak interactions is a well established evidence in Particle Physics. Nevertheless, the most recent combination of all $\mathrm{CP}$ violation measurements is found to be insufficient to explain the size of the matter anti-matter asymmetry present in the Universe. The large number of topantitop pair events produced in $p p$ colllisions at the Large Hadron Collider (LHC) provides a unique source of $b$-quarks that can be used to probe the $\mathrm{CP}$ violation in heavy-flavour mixing and decay.

This measurement [1] uses data corresponding to an integrated luminosity of $20.3 \mathrm{fb}^{-1}$ [2] from proton-proton collisions at a centre-of-mass energy of $\sqrt{s}=8 \mathrm{TeV}$ collected with the ATLAS [3] detector at the LHC at CERN. It selects events with exactly one lepton from a $W$-boson decay and at least four jets, one of which must be tagged with both a displaced-vertex $b$-tagging algorithm and the soft muon tagging (SMT) algorithm. Experimental ambiguity in determining the charge of the produced $b$-quark arises in $t \bar{t}$ pair production events when establishing if an SMT muon originated from the same- or different-top quark as the leptonically decaying $W$-boson. This can be resolved and corrected for as part of the unfolding procedure. An illustration of same- and different-top SMT muons is shown in Figure 1.

There are three classes of decay chains which produce two leptons of the same sign, given by the following left-hand side equations, and three classes of decay chains which produce two leptons of opposite sign, given by the following right-hand side equations [4]:

$$
\begin{aligned}
N_{b}: t \rightarrow \ell^{+} v(b \rightarrow \bar{b}) \rightarrow \ell^{+} \ell^{+} X & \widetilde{N}_{b}: t \rightarrow \ell^{+} v b \rightarrow \ell^{+} \ell^{-} X \\
N_{c}: t \rightarrow \ell^{+} v(b \rightarrow c) \rightarrow \ell^{+} \ell^{+} X & \widetilde{N}_{c}: t \rightarrow \ell^{+} v(b \rightarrow \bar{b} \rightarrow \bar{c}) \rightarrow \ell^{+} \ell^{-} X \\
N_{c \bar{c}}: t \rightarrow \ell^{+} v(b \rightarrow \bar{b} \rightarrow c \bar{c}) \rightarrow \ell^{+} \ell^{+} X & \widetilde{N}_{c \bar{c}}: t \rightarrow \ell^{+} v(b \rightarrow c \bar{c}) \rightarrow \ell^{+} \ell^{-} X .
\end{aligned}
$$

These processes are sensitive to CP violation in $B_{d, s}-\bar{B}_{d, s}$ mixing, semileptonic $b$ and $c$ decays and $b \rightarrow c$ transitions.

Experimentally, we can form charge asymmetries from the charge of the $W$-boson lepton and the soft muon from $b$ quark, which are sensitive to $\mathrm{CP}$ violation. The measurement focuses on determining relative differences in the probabilities of an initial $b$ or $\bar{b}$ to decay to either a positive or negative soft muon. These probabilities may be measured by considering the number of soft muons observed, where $N^{a b}$ represents the number of soft muons observed in conjunction with a $W$-boson lepton of charge $a$ and a soft muon of charge $b$. A total of four different probabilities are 
considered: $P\left(b \rightarrow \ell^{+}\right), P\left(\bar{b} \rightarrow \ell^{-}\right), P\left(b \rightarrow \ell^{-}\right), P\left(\bar{b} \rightarrow \ell^{+}\right)$.

A same sign (SS), and opposite sign (OS) asymmetry are formed from these probabilities:

$$
\begin{array}{rlrl}
A^{s s} & =\frac{P\left(b \rightarrow \ell^{+}\right)-P\left(\bar{b} \rightarrow \ell^{-}\right)}{P\left(b \rightarrow \ell^{+}\right)+P\left(\bar{b} \rightarrow \ell^{-}\right)} & A^{o s}=\frac{P\left(b \rightarrow \ell^{-}\right)-P\left(\bar{b} \rightarrow \ell^{+}\right)}{P\left(b \rightarrow \ell^{-}\right)+P\left(\bar{b} \rightarrow \ell^{+}\right)} \\
A^{s s}=\frac{\left(\frac{N^{++}}{N^{+}}-\frac{N^{--}}{N^{-}}\right)}{\left(\frac{N^{++}}{N^{+}}+\frac{N^{--}}{N^{-}}\right)} & A^{o s}=\frac{\left(\frac{N^{+-}}{N^{+}}-\frac{N^{-+}}{N^{-}}\right)}{\left(\frac{N^{+-}}{N^{+}}+\frac{N^{-+}}{N^{-}}\right)} .
\end{array}
$$

The CP asymmetries are related to fundamental CP violation parameters [4] via:

$$
\begin{aligned}
& A^{s s}=r_{b} A_{m i x}^{b \ell}+r_{c}\left(A_{d i r}^{b c}-A_{d i r}^{c \ell}\right)+r_{c \bar{c}}\left(A_{m i x}^{b c}-A_{d i r}^{c \ell}\right) \\
& A^{o s}=\widetilde{r}_{b} A_{d i r}^{b \ell}+\widetilde{r}_{c}\left(A_{m i x}^{b c}+A_{d i r}^{c \ell}\right)+\widetilde{r}_{c \bar{c}} A_{d i r}^{c \ell},
\end{aligned}
$$

where, in addition, we have defined: $r_{q} \equiv \frac{N_{q}^{++}+N_{q}^{--}}{N^{++}+N^{--}}$, with $q=b, c, c \bar{c}$ and $N_{b, c, c \bar{c}}^{ \pm \pm}$are the corresponding numbers of events coming from same-sign equations and $\widetilde{r}_{q}$ are the corresponding fractions of events for the decay chains defined in different-sign equations, respectively.

The number of events in each channel is given by [4]:

$$
N_{q}^{ \pm \pm}\left(N_{q}^{ \pm \mp}\right)=\sigma_{t \bar{t}} \mathscr{L} \mathrm{BR}(t \bar{t} \rightarrow b \bar{b} \ell v \text { had }) \varepsilon_{\mathrm{sel}} \varepsilon_{b}^{2} \varepsilon_{\mathrm{A}} \mathscr{B}_{q}
$$

where $q$ refers to the various same- and different- sign top processes, $\sigma_{t \bar{t}}$ is the top-pair production cross section, $\mathscr{L}$ is the integrated luminosity, $\operatorname{BR}(t \bar{t} \rightarrow b \bar{b} \ell v$ had $) \sim 0.30, \varepsilon_{\text {sel }}$ is the efficiency of selecting the lepton and the four jets, $\varepsilon_{b}$ is the $b$-tagging efficiency and $\varepsilon_{\mathrm{A}}$ is the $b$-charge association efficiency. Finally $\mathscr{B}_{q}$ is a factor which accounts for the relative probabilities of the top inclusive decay chains listed above for same sign and different sign leptons in the final states.

This analysis proceeds as follows:

1. The analysis first performs a "standard" $t \bar{t}$ lepton+jets events selection.

2. Events are selected in which a jet is $b$-tagged with both a displaced vertex based algorithm, called MV1, and a soft muon tagger algorithm, called SMT. For events containing more than one tagged jet, each SMT muon contributes to the measurement.

3. The inclusive $t \bar{t}$ cross section is measured to demonstrate that the data is well understood.

4. A kinematic likelihood algorithm, the KLFitter [5], is performed to fully reconstruct the $t \bar{t}$ system.

5. Charge asymmetry input distributions are measured in the data.

6. Background is subtracted and the data is unfolded to the fiducial volume.

7. The $\mathrm{CP}$ asymmetries are measured in data at the fiducial level.

8. The CP parameters are then determined via a fit. 

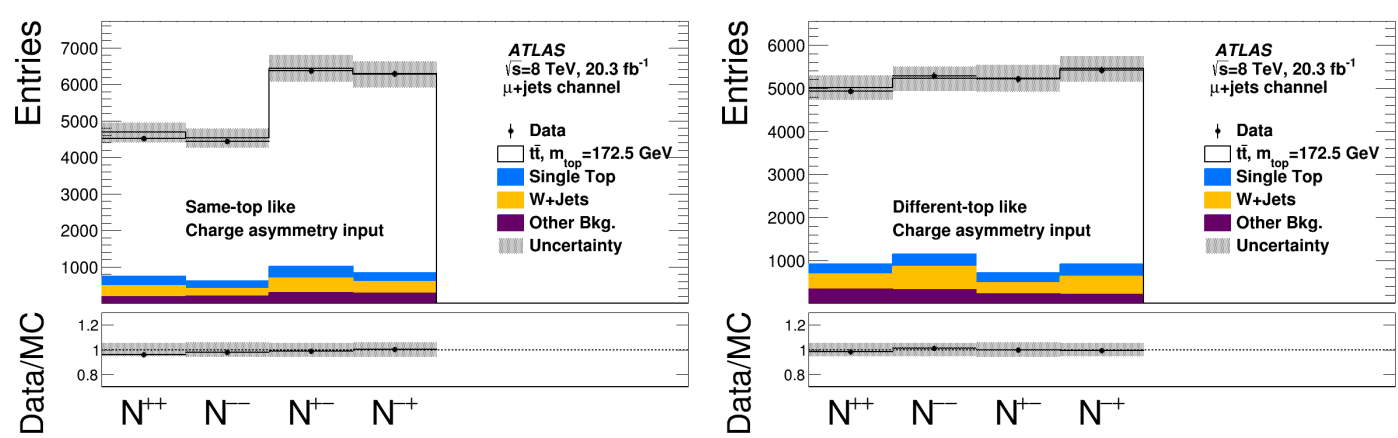

Figure 2: Same-top like (left) and different-top like (right) charge pairings distribution in the muon-channel. The hashed area represents all the experimental systematic uncertainties as well as the $b$-hadron production and hadron-to-muon branching ratio uncertainties. The lower panel of the distributions show the ratio of the data divided by the simulation [1].

\section{Measurement of charge asymmetries at $8 \mathrm{TeV}$}

The data are separated into same- and different-top-like SMT muons, as illustrated in Figure 1, by a kinematic likelihood fitter (KLFitter). The KLFitter places Breit-Wigner mass constraints on the top-quark and W-boson masses, and permutes reconstructed jets into each possible position in the leading-order parton representation of the $t \bar{t}$ system. If a reconstructed $b$-tagged jet is mapped to the KLFitter leptonic $b$-jet position then the SMT muon is considered to be same-top-like, whereas if the $b$-tagged jet is mapped to the KLFitter hadronic $b$-jet position then the SMT muon is considered to be different-top-like. The yield of SMT muons, that are designated as same-top-like is shown in Figure 2 (left) while those designated as different-top-like is shown in Figure 2 (right). For different-top-like SMT muons, the sign of the $W$-boson lepton has been reversed in order to consistently represent the charge of the $b$-quark at production in both the same- and different-top scenarios. The observed data are then combined and unfolded to the particle level.

The decay-chain fractions are obtained from simulation at the particle level. They can be used in conjunction with the observed charge asymmetries in order to extract the various $\mathrm{CP}$ asymmetries. The largest uncertainties in the decay-chain fractions come from the hadron-to-muon branching ratio and the parton shower. The data and MC predictions are compatible with zero and with the SM predictions, as shown in Table 1.

\section{Conclusions}

$\mathrm{CP}$ asymmetries are measured in $b$-hadron decays using top-pair events in $8 \mathrm{TeV}$ ATLAS data. Also a measurement of $A_{\mathrm{dir}}^{b c}$ is performed, which improves the existing $2 \sigma$ limit on $A_{\mathrm{dir}}^{c l}$ and provides an equivalent $2 \sigma$ limit on $A_{\mathrm{dir}}^{b c}$. All reported results are found to be consistent with the Standard Model. The largest uncertainty on all reported asymmetries is statistical. With the Run 2 ATLAS dataset, the statistical uncertainty will be smaller than the systematic uncertainties. In Run 1 the major source of systematic uncertainty was the additional radiation modelling, hence the aim is to reduce this systematic uncertainty in Run 2 analysis. 


\begin{tabular}{l|rr|cl} 
& Data $\left(10^{-2}\right)$ & MC $\left(10^{-2}\right)$ & Existing limits $(2 \sigma)\left(10^{-2}\right)$ & SM prediction $\left(10^{-2}\right)$ \\
\hline$A^{\mathrm{ss}}$ & $-0.7 \pm 0.8$ & $0.05 \pm 0.23$ & - & $<10^{-2}$ \\
$A^{\text {os }}$ & $0.4 \pm 0.5$ & $-0.03 \pm 0.13$ & - & $<10^{-2}$ \\
$A_{\text {mix }}^{b}$ & $-2.5 \pm 2.8$ & $0.2 \pm 0.7$ & $<0.1$ & $<10^{-3}$ \\
$A_{\text {dir }}^{b \ell}$ & $0.5 \pm 0.5$ & $-0.03 \pm 0.14$ & $<1.2$ & $<10^{-5}$ \\
$A_{\text {dir }}^{c \ell}$ & $1.0 \pm 1.0$ & $-0.06 \pm 0.25$ & $<6.0$ & $<10^{-9}$ \\
$A_{\text {dir }}^{b c}$ & $-1.0 \pm 1.1$ & $0.07 \pm 0.29$ & - & $<10^{-7}$
\end{tabular}

Table 1: Comparison of measurements of charge asymmetries and constraints on $\mathrm{CP}$ asymmetries, with MC simulation, existing experimental limits $[8,6]$ and SM predictions $[4,7,9]$. The latter two columns represent upper limits on the absolute values $|A|$. For $\mathrm{A}_{\text {mix }}^{b}$ the last two columns are determined using the prescription from Ref. [4], with inputs from the HFAG world average of $b$-hadrons properties [6] and either the world average [6] or the SM predictions [7] for $a_{s l}^{d}$ and $a_{s l}^{s}$ respectively.

\section{References}

[1] ATLAS Collaboration, Measurements of charge and CP asymmetries in b-hadron decays using top-quark events collected by the ATLAS detector in pp collisions at $\sqrt{s}=8 \mathrm{TeV}$, JHEP 02 (2017) 071.

[2] ATLAS Collaboration, Luminosity determination in pp collisions at $\sqrt{s}=8 \mathrm{TeV}$ using the ATLAS detector at the LHC, CERN-EP-2016-117 (2016).

[3] ATLAS Collaboration, The ATLAS Experiment at the CERN Large Hadron Collider, JINST 3 (2008) S08003.

[4] O. Gedalia, G. Isidori, F. Maltoni, G. Perez, M. Selvaggi and Y. Soreq, Top B Physics at the LHC, Phys. Rev. Lett. 110232002 2013, [arXiv:1212.4611].

[5] J. Erdmann et al., A likelihood-based reconstruction algorithm for top-quark pairs and the KLFitter framework, Nucl. Instrum. Meth. A 748 (2014) 18, [arXiv:1312.5595].

[6] Heavy Flavor Averaging Group (HFAG) collaboration, Y. Amhis et al., Averages of b-hadron, c-hadron and $\tau$-lepton properties as of summer 2014, [arXiv:1412.7515].

[7] M. Artuso, G. Borissov and A. Lenz, CP violation in the $B_{s}^{0}$ system, Rev. Mod. Phys. 88 (2016) 045002, [arXiv:1511.09466].

[8] D. Genon, On the origin of the DO like-sign dimuon charge asymmetry, Phys. Rev. D. 87074020.

[9] S. Bar-Shalom, G. Eilam, M. Gronau, J. L. Rosner, Second order direct CP asymmetry in $B_{s} \rightarrow X \ell v$, Phys. Lett. B 694 (2011) 374-379. 